\title{
NCI CTEP SDC CNS Neoplasm (Primary Tumor) Category Terminology
}

National Cancer Institute

\section{Source}

National Cancer Institute. NCI CT EP SDC CNS Neoplasm (Primary Tumor) Category

Terminology. NCl Thesaurus. Code C103010.

CNS Neoplasm (Primary Tumor) is an NCl Cancer Therapeutic Evaluation Program

(CTEP) Simplified Disease Classification (SDC) category used to organize cancer-related disease coding that harmonizes with and supports reporting based on the global standard Medical Dictionary for Drug Regulatory Reporting (MedDRA) terminology. 